\title{
Is it time to ban oxytocin for poor labour progress?
}

Dr Kate F. Walker*

MRCOG, PhD

Department of Obstetrics and Gynaecology, QMC, Nottingham, NG7 2UH

kate.walker@nottingham.ac.uk

01158231581

Dr George Bugg

MRCOG, MD

Department of Obstetrics and Gynaecology, Queens Medical Centre, Nottingham, NG7 2UH

George.Bugg@nuh.nhs.uk

01159249924

*Corresponding author

1500 words

\section{Abstract (150 words)}

Oxytocin causes myometrial contractions and prostaglandin release. It is commonly used as an intervention for women in established labour with slow progress. However recent large randomised trials of oxytocin for this indication have shown no difference in caesarean section rates associated with its use. This may be due to inadequate doses of oxytocin and small randomised studies have suggested high dose oxytocin may indeed reduce caesarean delivery. The results of a high quality large randomised trial of high versus low dose oxytocin for women with slow progress are keenly awaited. There is no evidence to support the concept of turning the oxytocin off once contractions have been established with the hormone although this certainly merits further investigation. Recent evidence of contemporary labour patterns indicate that our whole concept of slow progress in labour is fundamentally flawed.

Keywords

Oxytocin; syntocinon; labour progress; augmentation; failure to progress; first stage of labour

\section{Background}

Oxytocin, is a hormone produced by the hypothalamus and secreted by the posterior pituitary which acts on oxytocin receptors causing myometrial contractions and prostaglandin release in the decidua. 
Oxytocin receptor antagonists (e.g. atosiban) can be used for preterm labour to inhibit uterine contractions.

\section{Oxytocin for poor labour progress}

Oxytocin may be used for women in established labour with slow progress in an attempt to increase the frequency and power of uterine contractions and therefore cervical dilatation. A recent Cochrane review of 8 studies (1338 women) examined the effect of oxytocin use in low-risk women at term with slow progress in the active stage of labour. The review made two comparisons: firstly the use of intravenous oxytocin with either placebo or no treatment ( 3 trials, 138 women) and secondly the immediate use versus the delayed use ( $\geq$ one hour) of intravenous oxytocin ( 5 trials, 1200 women). For both comparisons there was no difference in caesarean delivery associated with oxytocin use for this indication. The only notable difference in the groups was that early use versus delayed use of oxytocin was associated with a two hour reduction in the time to delivery but not as a result of increased caesarean deliveries.

\section{What dose of oxytocin is sufficient?}

Are we simply not using high enough doses of oxytocin to see a difference in the groups?

A Cochrane review of 4 studies (644 women) comparing high dose (defined as starting dose and increment of $\geq 4 \mathrm{mU}$ per minute) with low-dose regimens (defined as starting dose and an increment of $<4 \mathrm{mU}$ per minute) of oxytocin found that high-dose regimens were associated with a reduction in the length of labour (mean difference 3.5 hours) and caesarean section rates (RR 0.62; $95 \% \mathrm{Cl} 0.44$ 0.86 ) and an increase in vaginal delivery ( $R R$ 1.35; $95 \% \mathrm{Cl} 1.13-1.62$ ) with no difference in uterine hyperstimulation or neonatal admission to a special care baby unit. Reported neonatal outcomes were limited and only provide limited reassurance of the safety for babies of high dose oxytocin. The HOLDS trial (planned sample size 1500 women) is a double blind RCT of standard dose oxytocin $(2 \mathrm{mU} / \mathrm{min}$ increasing every 30 minutes to a maximum $32 \mathrm{mU} / \mathrm{min})$ versus high dose regimen $(4 \mathrm{mU} / \mathrm{min}$ increasing every 30 minutes to a maximum of $64 \mathrm{mU} / \mathrm{min}$ ) which is currently recruiting and seeks to provide the definitive answer to the question (ISRCTN 99841044).

\section{Should we turn the oxytocin off once contractions are regular?}

Another plausible suggestions is that syntocinon could be used to initiate uterine contractions during active labour with slow progress but then be switched off. This is examined in a recent meta-analysis by Saccone et al which planned to examine both women in established labour undergoing augmentation for slow progress and women undergoing induction of labour but all nine trials identified, only included trials of women undergoing induction of labour. Perhaps surprisingly women in the intervention group which involved discontinuation of syntocinon once the active phase of labour was achieved, but reinstitution if slow progress (no cervical dilation in 2 hours) or inadequate uterine contractions (for $\geq 2$ hours) versus continuation of oxytocin until delivery found lower rates of uterine hyperstimulation (6.2\% versus $13 \%$; RR $0.53,95 \% \mathrm{Cl} 0.33-0.84$ ) and caesarean section $(9.3 \%$ versus $15 \%$; $\mathrm{RR} 0.64,95 \% \mathrm{Cl} 0.48-0.87$ ) associated with the intervention.

\section{Is our definition of slow progress in labour wrong fundamentally?}

What is acceptable progress in the active phase or first stage of labour? Secondly when does active labour start?

The standard definitions used by NICE for active labour is 'regular painful contractions and progressive cervical dilatation from $4 \mathrm{~cm}$ ' and for slow progress in active labour is cervical dilatation of less than $2 \mathrm{~cm}$ in 4 hours for first labours. This is largely based on work from 50 years ago on labour progress in a medicalised setting. While the introduction of partograms is an important safety net to prevent 
the maternal and neonatal morbidity and mortality associated with obstructed labour, some have argued that the strict parameters used to define adequate progress in labour pathologise a normal physiological progress. In 2010 Zhang et al re-examined labour patterns using contemporary labour data and found that progress from 4 to $6 \mathrm{~cm}$ was much slower than previously described leading the American College of Obstetricians and Gynaecologists to recommend that active labour should not be diagnosed until $6 \mathrm{~cm}$ dilatation if there are no fetal or maternal concerns. Given that the leading reason for primary caesarean delivery in the US is slow labour progress this could have a dramatic effect on CS rates in the US. Zhang's extensive work in this area, summarised succinctly in a narrative review by Karacam et al has shown us that labour progress is not linear, but rather occurs in a stepwise fashion with fluctuating periods of "acceleration and quiescence".

\section{Alternatives to oxytocin}

Titrated oral misoprostol, a prostaglandin analogue, widely used in reproductive health, has been researched as an alternative to oxytocin for slow labour progress. A Cochrane review of two trials (581 women) with different misoprostol regimes (20mcg repeated hourly, $75 \mathrm{mcg}$ repeated four hourly) found inadequate maternal and neonatal adverse outcome reporting to determine safety of misoprostol for this indication. It also found conflicting results where women in the lower dose group (20 mcg) had a lower incidence of tachysystole than those in the oxytocin group and conversely women in the higher dose group $(75 \mathrm{mcg})$ had a higher incidence of hyperstimulation than those in the oxytocin group. It concluded that there was insufficient evidence of the safety and efficacy of misoprostol to recommend its use for this indication.

\section{Conclusion}

Oxytocin used for slow labour progress has a modest effect on the duration of labour but not reduce caesarean section rates. This finding could be explained by the testing of inadequate doses of oxytocin used in trials in this area. The results of a large randomised trial of high versus low dose oxytocin are eagerly awaited. The questions: can oxytocin be used to increase contractions for slow labour and then turned off; can oral misoprostol be used to augment labour, merit further research. Research into contemporary labour patterns indicates that active labour starts at $6 \mathrm{~cm}$, not $4 \mathrm{~cm}$, implementing this change could result in a reduction in the diagnosis of slow labour, requirement for oxytocin augmentation and emergency caesarean sections for slow labour progress.

\section{Further Reading List}

1. Bugg GJ, Siddiqui F, Thornton JG: Oxytocin versus no treatment or delayed treatment for slow progress in the first stage of spontaneous labour. Cochrane Db Syst Rev 2013(6).

2. Kenyon $S$, Tokumasu $H$, Dowswell $T$, Pledge $D$, Mori R: High-dose versus low-dose oxytocin for augmentation of delayed labour. Cochrane Db Syst Rev 2013(7).

3. Saccone G, Ciardulli A, Baxter JK, Quinones JN, Diven LC, Pinar B, Maruotti GM, Martinelli P, Berghella V: Discontinuing Oxytocin Infusion in the Active Phase of Labor A Systematic Review and Meta-analysis. Obstet Gynecol 2017, 130(5):1090-1096.

4. Zhang J, Landy HJ, Branch DW, Burkman R, Haberman S, Gregory KD, Hatjis CG, Ramirez MM, Bailit JL, Gonzalez-Quintero VH et al: Contemporary Patterns of Spontaneous Labor With Normal Neonatal Outcomes. Obstet Gynecol 2010, 116(6):1281-1287.

5. Karacam Z, Walsh D, Bugg GJ: Evolving understanding and treatment of labour dystocia. Eur J Obstet Gyn R B 2014, 182:123-127. 